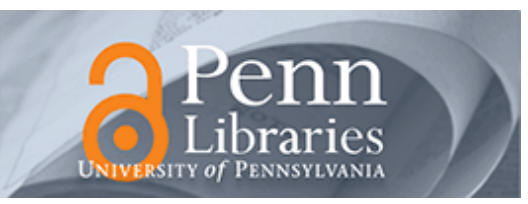

University of Pennsylvania

ScholarlyCommons

$11-1-2010$

\title{
High Frequency Piezoelectric Resonant Nanochannel for Bio- Sensing Applications in Liquid Environment
}

\author{
Chiara Zuniga \\ University of Pennsylvania, zunigac@seas.upenn.edu \\ Matteo Rinaldi \\ University of Pennsylvania, rinaldim@seas.upenn.edu \\ Gianluca Piazza \\ University of Pennsylvania, piazza@seas.upenn.edu
}

Follow this and additional works at: https://repository.upenn.edu/ese_papers

Part of the Electrical and Computer Engineering Commons

\section{Recommended Citation}

Chiara Zuniga, Matteo Rinaldi, and Gianluca Piazza, "High Frequency Piezoelectric Resonant Nanochannel for Bio-Sensing Applications in Liquid Environment", . November 2010.

Suggested Citation:

Zuniga, Chiara; Rinaldi, Matteo; Piazza, Gianluca; , "High frequency Piezoelectric Resonant Nanochannel for biosensing applications in liquid environment," Sensors, 2010 IEEE , vol., no., pp.52-55, 1-4 Nov. 2010

C2010 IEEE. Personal use of this material is permitted. However, permission to reprint/republish this material for advertising or promotional purposes or for creating new collective works for resale or redistribution to servers or lists, or to reuse any copyrighted component of this work in other works must be obtained from the IEEE.

This paper is posted at ScholarlyCommons. https://repository.upenn.edu/ese_papers/590

For more information, please contact repository@pobox.upenn.edu. 


\title{
High Frequency Piezoelectric Resonant Nanochannel for Bio-Sensing Applications in Liquid Environment
}

\author{
Abstract \\ This paper reports on the first demonstration of a $457 \mathrm{MHz}$ AIN Piezolectric Resonant Nanochannel \\ (PRN) for biosensing applications in liquid environment. A novel process consisting of 7 lithographic \\ steps was developed to fabricate the PRN. The new resonant device shows an unchanged value of the \\ electromechanical coupling, $k_{t}{ }^{2}$ (about $0.8 \%$ ), whether the channel is filled with air or water and a quality \\ factor, $Q$, in liquid of approximately 170 . The value of $k_{t}{ }^{2}$ and $Q$ are respectively about 2.7 and 2 times the \\ ones recorded for conventional laterally vibrating AIN Contour Mode Resonant Sensors (CMR-Ss) \\ submerged in water. Overall, these results translate in a $\sim 5$ fold enhancement in the figure of merit $\left(k_{t}{ }^{2}\right.$. \\ $Q$ product) of the resonant device when operated in liquid and simultaneously permit the efficient delivery \\ of ultra-low concentrations of fluid samples directly on the surface of the sensor. \\ Disciplines \\ Electrical and Computer Engineering | Engineering \\ Comments \\ Suggested Citation: \\ Zuniga, Chiara; Rinaldi, Matteo; Piazza, Gianluca; , "High frequency Piezoelectric Resonant Nanochannel \\ for bio-sensing applications in liquid environment," Sensors, 2010 IEEE , vol., no., pp.52-55, 1-4 Nov. 2010 \\ C2010 IEEE. Personal use of this material is permitted. However, permission to reprint/republish this \\ material for advertising or promotional purposes or for creating new collective works for resale or \\ redistribution to servers or lists, or to reuse any copyrighted component of this work in other works must \\ be obtained from the IEEE.
}




\title{
High Frequency Piezoelectric Resonant Nanochannel for Bio-Sensing Applications in Liquid Environment
}

\author{
Chiara Zuniga, Matteo Rinaldi and Gianluca Piazza \\ Department of Electrical and Systems Engineering \\ University of Pennsylvania \\ Philadelphia, PA, USA \\ \{zunigac, rinaldim, piazza\}@seas.upenn.edu
}

\begin{abstract}
This paper reports on the first demonstration of a $457 \mathrm{MHz}$ AIN Piezolectric Resonant Nanochannel (PRN) for biosensing applications in liquid environment. A novel process consisting of 7 lithographic steps was developed to fabricate the PRN. The new resonant device shows an unchanged value of the electromechanical coupling, $k_{t}{ }^{2}$ (about $0.8 \%$ ), whether the channel is filled with air or water and a quality factor, $Q$, in liquid of approximately 170. The value of $k_{t}^{2}$ and $Q$ are respectively about 2.7 and 2 times the ones recorded for conventional laterally vibrating AIN Contour Mode Resonant Sensors (CMR-Ss) submerged in water. Overall, these results translate in a $\sim 5$ fold enhancement in the figure of merit $\left(k_{t}^{2}-Q\right.$ product) of the resonant device when operated in liquid and simultaneously permit the efficient delivery of ultra-low concentrations of fluid samples directly on the surface of the sensor.
\end{abstract}

\section{INTRODUCTION}

Lab-on-chip systems are considered an interesting engineering solution that will address issues in public health and welfare by increasing analysis throughput and dramatically reducing reagent cost.

An essential component in the development and miniaturization of lab-on-chip systems is the bio-detector. Acoustic gravimetric sensors, such as Quartz Crystal Microbalances (QCMs), have shown great potentials when employed as bio-detectors [1]. The main advantage of this category of sensors compared to other technologies such as conductance-based sensors (e.g. Chem-FET), or optical sensors, lies in the fact that they use frequency as the output variable, which is one of the physical quantities that can be monitored with the highest accuracy [2]. However, because of their size, QCMs fail to represent a viable solution for labon-chip applications that require miniaturized sensors integrated in large arrays.

In recent years, miniaturized devices based on micro and nanoelectromechanical systems (MEMS/NEMS) technology have emerged as a viable response to the need for compact, inexpensive and integrable resonant sensors. Their reduced mass allows them to achieve unprecedented levels of

This work was supported by the National Consortium for MASINT Research (NCMR), the National Science Foundation (NSF) and the Nanotechnology Institute (NTI). resolution [3] and makes them suitable for detecting minuscule concentrations of target analyte in bio-samples.

However, when M/NEMS resonant sensors are required to operate in a liquid environment (typical of bio-applications), additional challenges need to be overcome in order to achieve the same levels of performance shown in air.

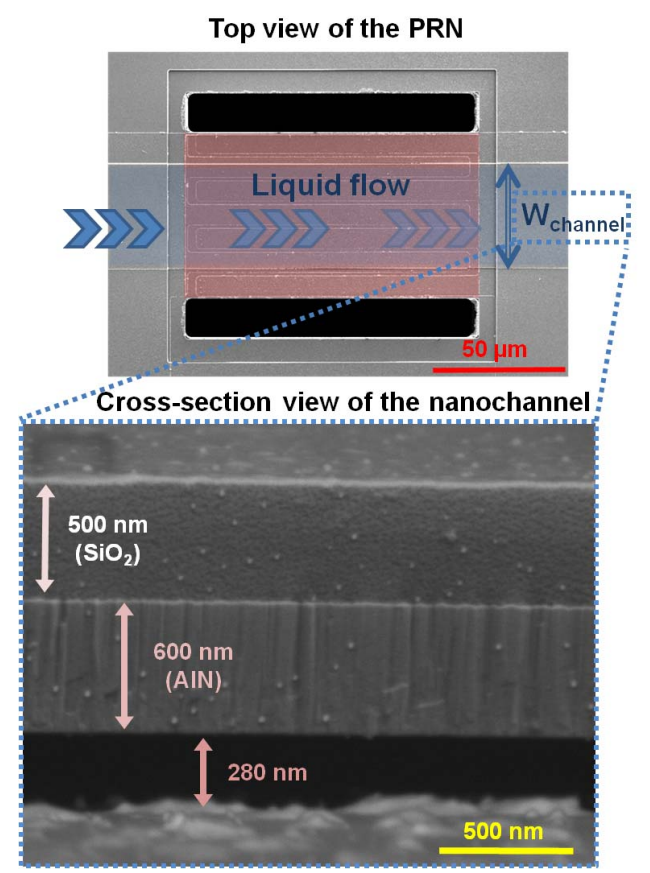

Figure 1. Scanning Electron Microscopy (SEM) images of the PRNsensor and the nanochannel cross-section. It is worth noting that the silicon dioxide layer is not present on top of the resonant section (solely formed by AlN), but exclusively on the portion of the channel that leads to the inlet and oulet of the resonator.

The operation of acoustic gravimetric sensors in liquid environment is in fact degraded by the viscous force acting on the resonator surface, which causes energy radiation in the surrounding media and consequently decreases the device 
quality factor, $Q$, and its frequency resolution. In addition the immersion of the resonant device in the liquid media renders its transduction more challenging and less efficient, which translates in the need of cumbersome and power consuming actuation and readout techniques. Although there have been demonstrations of bio-detection via mass sensing in miniaturized resonators such as cantilever beams [4], these prototypes have experienced reduced performance compared to operation in air or vacuum, and suffered from the lack of an adequate fluidic system for the delivery of the bio-samples in a small volume and in direct contact with the surface of the resonant device.

The integration of a microfluidic channel inside the body of a resonant cantilever beam sensor [3] has been proposed as a solution to simultaneously address the issue of viscous damping and realizing a compact delivery system that reduces sample volume. Although highly effective in maintaining unaltered mechanical performances, these hollow cantilever beams required a high DC voltage (as high as $120 \mathrm{~V} \mathrm{[3])} \mathrm{for}$ their electrostatic actuation and a cumbersome and power inefficient optical readout. In addition, the reduced frequency of operation (few 100s of $\mathrm{KHz}$ ) limited the device sensitivity and ultimate resolution for certain applications.

Recently, the capability to fabricate higher frequency $(100 \mathrm{MHz}-10 \mathrm{GHz})$ [5] resonant sensors with ultra-low values of limit of detection (LOD) in air [6, 7] has been demonstrated employing the Nanoscaled AlN Contour Mode Resonant Sensor (CMR-S) technology.

In this work, for the first time, a Piezoelectric Resonant Nanochannel Sensor is proposed as an innovative solution for the realization of resonant bio-sensor devices with high frequency of operation, reduced viscous damping, improved transduction efficiency and effective delivery of the biosample solely on the active sensing surface of the device. By transforming the resonant body of the CMR into a laterally vibrating nanochannel, the thickness of the viscous layer as well as the total amount of liquid in contact with the resonator surface has been reduced. Therefore, the new device achieves a 5 fold enhancement in the figure of merit $\left(k_{t}^{2}-Q\right.$, product of the electromechanical coupling and resonator $Q$ ) with respect to a conventional CMR-S and results in an unchanged $k_{t}^{2}$ (approximately $0.8 \%$ ) with respect to the measurement in air and a $Q$ in fluid of approximately 170 . Furthermore, the novel layout permits the delivery of the fluid solely and in close proximity of the sensing surface (which can also be doubled with respect to the conventional CMR-S), therefore resulting in a greatly improved device-sample interaction.

\section{DESIGN}

An efficient method for the delivery of the bio-sample onto the resonant element is ultimately of crucial importance in determining the overall performance of a resonant biosensor. In fact, if the resonator were to be immersed in a large volume of the fluid sample, it would not be statistically able to interact with some of the very low concentration analytes contained in the sample itself. Therefore, a major fraction of the analyte would be exhausted without contributing to the detection process [8].
In this perspective, the implementation of a high surface to volume ratio resonant nanochannel represents the optimum design solution, since it allows confining a small sample volume $(\mathrm{pL})$ in a thin layer proportional to the penetration depth of the acoustic wave (i.e. closely in contact with the entire vibrating structure), reducing viscous drag and maximizing the sample-device interaction.

Simultaneously, the design choice of forming a resonant nanochannel solves another issue related to the transduction efficiency of nanoscale structures in liquids. The PRN confines the electric energy (necessary for actuation) in the piezoelectric body of the device minimizing energy leak into the fluid. In fact, if a conventional CMR-S were simply immersed in a large volume of fluid, most of the electrical energy would be confined in the fluid rather than the resonator body due to the higher dielectric constant of the fluid with respect to the AlN piezoelectric film.

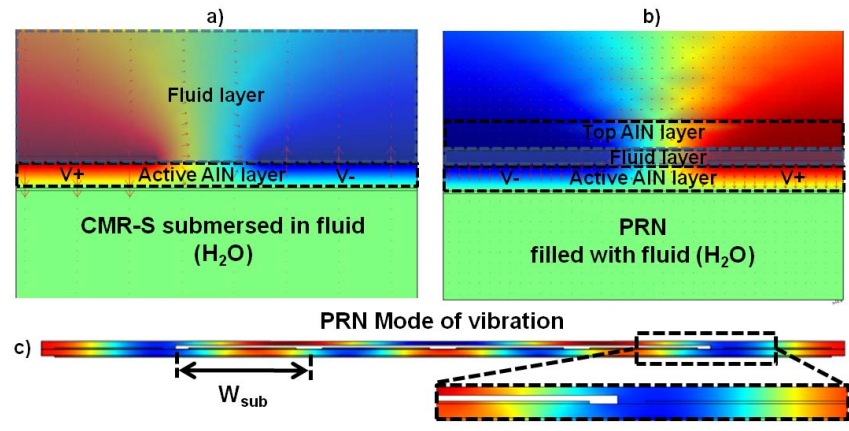

Figure 2. (a) Zoomed in view (two adjecent electrodes) of FEM simulation results for the electric potential (colors) and electric field (arrows) distribution in a conventional CMR-S. ((b) and a Piezoelectric Resonant Nanochannel (b). (c) displays the mode of vibration of the PRN, highlighting that the entire body of the channel is actually vibrating at the prescribed resonance frequency (set by $W_{\text {sub }}$ ).

The beneficial effect that the design of a resonant nanochannel has on the confinement of electrical energy in the resonant device and consequently on its electromechanical coupling coefficient is confirmed by $2 \mathrm{D}$ electrostatic Finite Element Method (FEM) analysis performed using COMSOL Multiphysics 3.5 - Electrostatic AC/DC Module. As shown in Table I, the presence of fluid (set to be water in the analysis) is such that most of the electrical energy is confined in the fluid rather than the resonator when a conventional CMR-S is used. When instead the PRN is employed, most of the electrical energy is effectively stored in the piezoelectric (active) body of the device rather than the liquid media.

This beneficial effect on the device electrical performance can be alternatively seen as a reduction in the parasitic capacitance introduced by the liquid media. In fact, the presence of water introduces a large parasitic capacitance, $C_{p}$ $(\sim 680 \mathrm{fF})$, in parallel to the device geometrical capacitance, $C_{0}$, which, as highlighted in [9], negatively affects the $k_{t}^{2}$ of the device $\left(k_{t}^{2} \propto C_{m} / C_{0}\right)$. It is possible to observe (Table I) that, when the PRN is employed, the value of such parasitic parallel capacitance, $C_{p}$, is significantly reduced $\left(C_{p} \sim 38 \mathrm{fF}\right)$ and negligible if compared to the device capacitance, $C_{0}$. 
Based on these considerations, the PRN is designed to have a nanoscaled channel depth of $280 \mathrm{~nm}$. The device is formed by two AlN layers, $500 \mathrm{~nm}$ and $600 \mathrm{~nm}$ thick respectively, separated by an air gap of $280 \mathrm{~nm}$.

TABLE I. COMSOL FEM SIMULATIONS OF DEVICE ELECTROSTATICS

\begin{tabular}{|c|c|c|c|}
\hline FEM Simulations & CMR-S & $\begin{array}{c}\text { CMR-S } \\
\text { w/ top AIN }\end{array}$ & PNR-S \\
\hline $\boldsymbol{C}_{\boldsymbol{0}}$ - Geometrical & $244 \mathrm{fF}$ & $267 \mathrm{fF}$ & $260 \mathrm{fF}$ \\
\hline $\begin{array}{c}\boldsymbol{C}_{\boldsymbol{p}}-\text { Parasitic in } \\
\mathbf{H}_{\mathbf{2}} \mathbf{O}\end{array}$ & $682 \mathrm{fF}$ & $173 \mathrm{fF}$ & $38 \mathrm{fF}$ \\
\hline $\begin{array}{c}\boldsymbol{C}_{\text {tot }} \text { - Total } \\
\text { capacitance in } \mathbf{H}_{2} \mathbf{O}\end{array}$ & $926 \mathrm{fF}$ & $440 \mathrm{fF}$ & $298 \mathrm{fF}$ \\
\hline $\begin{array}{c}\text { Energy density } \\
\text { stored in device }\end{array}$ & $\begin{array}{c}1.01 \\
\mathrm{~nJ} / \mathrm{m} \cdot \mathrm{V}^{2}\end{array}$ & $\begin{array}{c}1.01 \\
\mathrm{~nJ} / \mathrm{m} \cdot \mathrm{V}^{2}\end{array}$ & $1.04 \mathrm{~nJ} / \mathrm{mV}^{2}$ \\
\hline $\begin{array}{c}\text { Energy density } \\
\text { dissipated in } \mathbf{H}_{2} \mathbf{O}\end{array}$ & $\begin{array}{c}1.74 \\
\mathrm{~nJ} / \mathrm{m} \cdot \mathrm{V}^{2}\end{array}$ & $\begin{array}{c}0.67 \\
\mathrm{~nJ} / \mathrm{m} \cdot \mathrm{V}^{2}\end{array}$ & $0.174 \mathrm{~nJ} / \mathrm{m} \cdot \mathrm{V}^{2}$ \\
\hline
\end{tabular}

The first AlN layer (active layer, $500 \mathrm{~nm}$ ) is actively transduced (piezoelectrically) to generate vibration in the PRN, while the second AIN layer (structural layer, $600 \mathrm{~nm}$ ) is used to form the top surface of the resonant nanochannel. Similarly to conventional CMR-S [6], the resonance frequency of the PRN is approximately set by the width, $W_{s u b}$, of an individual device finger (Fig. 2-c) and the equivalent acoustic velocity of the material stack forming the resonator. The active AlN layer of the PRN of this work is formed of 6 fingers $(n=6)$ whose width, $W_{\text {sub }}$, is set to be $10 \mu \mathrm{m}$. This configuration translates in a frequency of operation of 457 $\mathrm{MHz}(\sim 1000 \mathrm{X}$ higher than the one achieved by Suspended Nanochannel Resonators [3]). The total width of the PRN is $60 \mu \mathrm{m}$ with an effective nanochannel width of $40 \mu \mathrm{m}$. The total length of the channel (from inlet to outlet) is $4.1 \mathrm{~mm}$. Therefore, the nanochannel occupies a surface area of 60,000 $\mu \mathrm{m}^{2}$ and an overall volume of $46 \mathrm{pL}$. The device active region (which undergoes mechanical vibrations) occupies a surface area of $4,800 \mu^{2}$ and an overall volume of only $1.34 \mathrm{pL}$.

\section{FABRICATION PROCESS}

The wafer level integration of the $280 \mathrm{~nm}$ AlN resonantnanochannel was realized through a 7 mask fabrication (Fig.3) by adding 3 lithographic steps to the standard process developed for CMR-S [6].

Optical lithography was first performed for the definition of the bottom floating Pt plate $(50 \mathrm{~nm})$ sputtered on a high resistivity $\mathrm{Si}$ substrate. This step was followed by sputter deposition of c-axis piezoelectric AIN thin (500nm) film (Fig.3-a). This first layer of the AlN represents the active piezoelectric film that is sandwiched between the two metal layers; however, the entire structure forming the PRN is excited into lateral vibration as shown in Fig.2-c.

The top Pt layer $(50 \mathrm{~nm})$ was deposited by evaporation and patterned by a lift-off process (Fig.3-b). A very thin film $(\sim 15 \mathrm{~nm})$ of Plasma Enhanced Chemical Vapor Deposition
(PECVD) deposited silicon dioxide $\left(\mathrm{SiO}_{2}\right)$ was placed onto the device top surface to enhance the adhesion between $\mathrm{Pt}$ and the subsequent layer of amorphous silicon (a-Si).

a-Si (280 nm thick) was then evaporated and patterned through reactive ion etching (RIE) in $\mathrm{SF}_{6}$ chemistry to define the shape of the nanochannel (Fig.3-c). A second layer of AlN (600 nm thick) was sputtered on the patterned sacrificial layer to form the top encapsulation of the resonant nanofluidic channel (Fig.3-d). Wet etch in phosphoric acid $\left(\mathrm{H}_{3} \mathrm{PO}_{4}\right)$ at $150{ }^{\circ} \mathrm{C}$ was employed to open vias in the top AlN layer in order to access the pads for the electrical connections to the device (Fig.3-e).

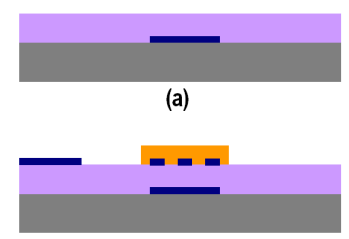

(c)

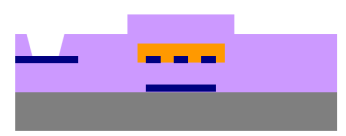

(e)

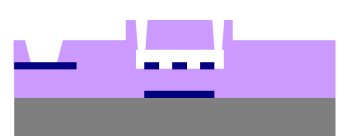

(g)

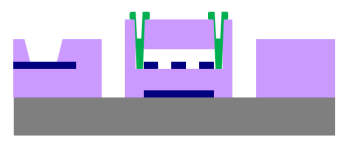

(i)

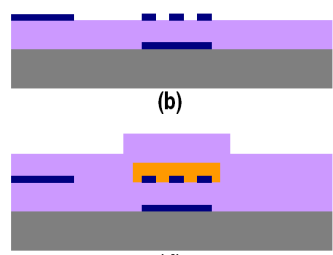

(d)

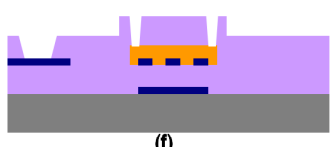

(f)

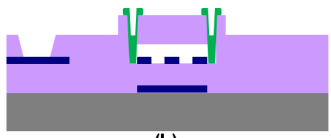

(h)

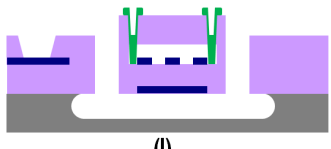

(I) $\square \mathrm{Si} \square \mathrm{Pt} \square$ AIN $\square \mathrm{a}-\mathrm{Si} \square \mathrm{SiO}_{2}$

Figure 3. Fabrication process of the PRN. The process requires 3 additional lithographic steps with respect to the standard fabrication of CMR.

In order to remove the amorphous silicon sacrificial layer and to form the inlet/outlet openings of the nanochannel, 100 $\mathrm{x} 100 \mu \mathrm{m}$ etch holes were defined on the top AIN layer at the extremities of the channel by inductively coupled plasma (ICP) dry etching. Simultaneously, small holes $(5 \times 10 \mu \mathrm{m})$ were periodically defined along the length of the channel in order to more efficiently remove the amorphous silicon (Fig.3-f).

The nanochannel was released by dry etching of the amorphous Silicon in $\mathrm{XeF}_{2}$ (Fig.3-g). Subsequently the small holes (used to etch it) were sealed through a $500 \mathrm{~nm}$ thick $\mathrm{SiO}_{2}$ layer deposited by PECVD. This layer of oxide was removed (by $\mathrm{CH}_{6}$ chemistry $\mathrm{RIE}$ ) only from the body of the device, the pads for the electrical connections as well as the inlet/outlet of the nanochannel (Fig.3-h).

Lastly, the body of the resonator was defined by ICP dry etching of the two AIN layers and the PRN was released from the substrate by $\mathrm{XeF}_{2}$ isotropic dry etching of silicon as shown in Fig.3-i,1. 


\section{EXPERIMENTAL RESULTS}

The performance of the $457 \mathrm{MHz}$ AlN Piezolectric Resonant Nanochannel were evaluated by testing the device in an RF probe station and recorded by an Agilent PNA N5230A network analyzer. Its electrical response with and without any liquid filling the channel was characterized and its equivalent electrical parameters were extracted by fitting the admittance curves to a MBVD model [10].

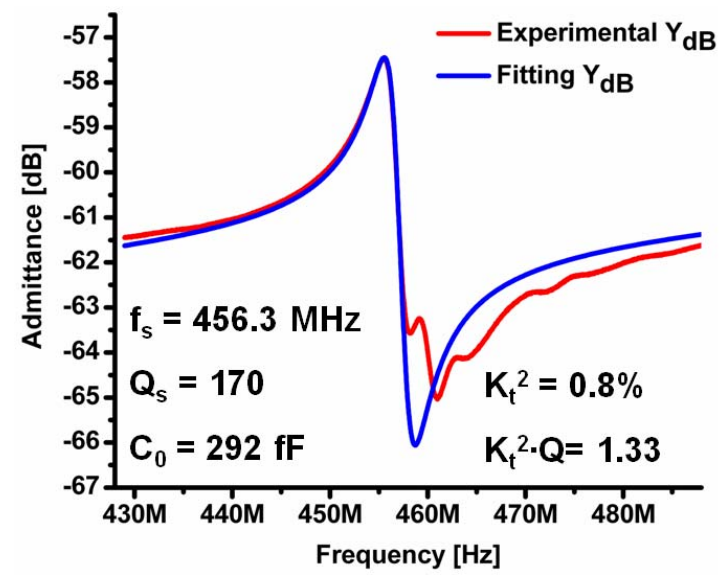

Figure 4. Electrical response of the $457 \mathrm{MHz} P R N$ with de-ionized water filling the nanochannel and in ambient conditions.

In order to test the device response in a viscous environment, a drop of $\sim 1 \mu \mathrm{L}$ of de-ionized water was dispensed through a micropipette on top of one of the inlets of the nanochannel: as a result, part of the drop $(46 \mathrm{pL}$ is the total volume of the channel) was sucked inside the nanofluidic channel by capillary forces and a difference in the density of the viscous fluid (going from air to water) was recorded by the device. The device experienced a drop in $Q$ (with respect to air) because of the additional damping introduced by the liquid media confined in its body. A quality factor in liquid equal to 170 was achieved proving that delivering a reduced thickness of the viscous layer as well as a smaller total amount of liquid in contact with the resonator surface determines a $2 \mathrm{X}$ enhancement in the device quality factor. The extracted experimental data are summarized in Table II, where the PRN is compared to a CMR device of identical total thickness $(1.1 \mu \mathrm{m})$ that was fabricated at the same time side to side to the PRN, but without the airgap forming the resonant nanochannel.

Despite the decrease in quality factor, the electromechanical coupling coefficient of the PRN remained unaltered and equal to $0.8 \%$ proving that the confinement of the fluid is advantageous in reducing the parasitic capacitance in parallel with the device and improving the resonator transduction efficiency. This result directly relates to a better confinement of the electric energy in the AlN body of the resonator and is in line with the FEM predictions.
TABLE II. SUMMARY OF EXPERIMENTAL RESULTS

\begin{tabular}{|c|c|c|c|c|c|c|}
\hline \multicolumn{2}{|c|}{} & $\begin{array}{c}\mathbf{f}_{\mathbf{0}} \\
{[\mathbf{M H z}]}\end{array}$ & $\mathbf{Q}$ & $\mathbf{C}_{\mathbf{0}}$ & $\mathbf{k}_{\mathbf{t}}{ }^{2}$ & $\mathbf{k}_{\mathbf{t}}{ }^{2}{ }^{*} \mathbf{Q}$ \\
\hline \multirow{2}{*}{ PRN } & Air & 457.9 & 1082 & 278 & $0.83 \%$ & 9.13 \\
\cline { 2 - 7 } & $\mathrm{H}_{2} \mathrm{O}$ & 456.3 & 170 & 292 & $0.80 \%$ & 1.33 \\
\hline $\begin{array}{c}\text { CMR-S } \\
\text { w/ top } \\
\text { AIN }\end{array}$ & Air & 460.7 & 961 & 306.5 & $0.97 \%$ & 9.36 \\
\cline { 2 - 7 } & $\mathrm{H}_{2} \mathrm{O}$ & 458.6 & 90 & 478 & $0.29 \%$ & 0.26 \\
\hline
\end{tabular}

Differently, the conventional CMR suffered from a more damped response when immersed in liquid ( $Q$ of 90), and a less efficient transduction $\left(k_{t}^{2} \sim 0.29 \%\right)$ due to the large parasitic capacitance introduced by the liquid media.

\section{CONCLUSIONS}

In this work, for the first time, a high frequency (457 MHz) Piezoelectric Resonant Nanochannel for operation in liquid media was demonstrated. This new device shows an unchanged value of $k_{t}^{2}(0.8 \%)$ whether the channel is filled with air or water and a $Q$ in liquid of approximately 170 , which translates in a $5 \mathrm{X}$ enhancement in performance with respect to a conventional CMR-S submerged in liquid media. This innovative solution for the realization of resonant biosensor devices combines a high frequency of operation (1000 X higher than cantilever beams) and the capability to employ efficient on chip actuation and sensing of the device, therefore enabling the use of a compact and low power electronic readout. Additionally, because of the unique capability of arraying large number of PRNs with different frequencies of operation in a small footprint, this novel technology lays the foundation towards the demonstration of large arrays of Piezoelectric Resonant Nanochannels for highly multiplexed bio-sensing applications that require high throughput and reduced sample volume.

\section{REFERENCES}

[1] L. Arce, M. Zougagh, C. Arce, A. Moreno, A. Rios, M. Valcarcel, Biosensors and Bioelectronics 22 (2007) pp. 3217-3223.

[2] J. R. Vig, L. Filler, Y. Kim, J. Microelectromech. Syst., vol. 5, no. 2, pp. 131-137, 1996.

[3] Jungchul Lee, Wenjiang Shen, Kris Payer, Thomas P. Burg, and Scott R. Manalis, Nano Lett. 2010, 10, 2537-2542.

[4] M. Yue, J. C. Stachowiak, H. Lin, R. Datar, R. Cote and A. Majumdar, Nano Lett., 2008, 8 (2), pp 520-524.

[5] M. Rinaldi, C. Zuniga, and G. Piazza, Proc. 22nd IEEE International Conference on Micro Electro Mechanical Systems (MEMS 2009), Sorrento, Italy, Jan. 2009, pp. 916-919.

[6] M. Rinaldi, B. Duick, C. Zuniga, C. Zuo and G. Piazza , Proc. 23rd IEEE International Conference on Micro Electro Mechanical Systems (MEMS 2010), pp. 132-135.

[7] M. Rinaldi, C. Zuniga, C. Zuo and G. Piazza, Proc. Solid-State Sensors, Actuators, and Microsystems Workshop (Hilton Head 2010), Jun. 2010, pp. 471-474.

[8] G. Wingqvist, J. Bjurstrom, A.-C. Hellgren, I. Katardjiev, Sensors and Actuators B 127 (2007) 248-252.

[9] M. Rinaldi, C. Zuniga, C. Zuo and G. Piazza, IEEE Transactions on Ultrasonics, Ferroelectrics, and Freq. Control, Vol.57 No.1, Jan. 2010, pp 82-87.

[10] J.D. Larson, P.D. Bradley, S. Wartenenberg, and R.C. Ruby, Proc. IEEE Ultrason. Symp., Oct. 2000, pp.863-868. 